\title{
Análogos das prostaglandinas diminuem a sensibilidade do teste provocativo da ibopamina no glaucoma
}

\author{
Prostaglandin analogues reduce the ibopamine provocative test specificity in glaucoma
}

\author{
Leopoldo Magacho ${ }^{1}$ \\ Marcelus Layguel Costa ${ }^{2}$ \\ Francisco Eduardo Lima ${ }^{3}$ \\ Bernardo Magacho ${ }^{4}$ \\ Marcos Pereira de Ávila ${ }^{5}$
}

\begin{tabular}{l} 
RESUMO \\
\hline Objetivo: Avaliar o desempenho do teste provocativo da ibopamina em \\
pacientes com glaucoma usuários de drogas hipotensoras. Métodos: \\
Pacientes glaucomatosos foram recrutados do Centro de Referência em \\
Oftalmologia (CEROF) da Universidade Federal de Goiás, e suas drogas \\
hipotensoras em uso registradas. Indivíduos normais foram amigos e \\
parentes dos pacientes. A seguir, foram instiladas duas gotas de ibopamina \\
$2 \%$ com intervalo de 5 minutos. A pressão intra-ocular (Pio) foi medida \\
previamente, e após 30,60 e 180 minutos. No nosso estudo, o teste da \\
ibopamina foi considerado positivo quando a pressão intra-ocular exce- \\
deu4mmHg em pelo menos uma das medidas. Resultados: Cinquentaeoito \\
olhos de 58 indivíduos (38 glaucomatosos e 20 normais) foram incluídos \\
no estudo. O aumento da pressão intra-ocular foi maior nos pacientes com \\
glaucoma aos 30,60 e 180 minutos (p<0,001 em todas as ocasiões). O \\
aumento da pressão intra-ocular foi estatisticamente maior aos 30 minutos \\
nos indivíduos que não utilizavam análogos das prostaglandinas (p=0,01). \\
A sensibilidade do teste foi de $68 \%$ (87\% se excluirmos pacientes em uso \\
de análogos das prostaglandinas), e a especificidade de 95\%. Conclusão: \\
O teste provocativo da ibopamina $2 \%$ pode ser ferramenta auxiliar no \\
diagnóstico do glaucoma. Apesar da pequena amostra estudada, sugere- \\
se que olhos em uso de análogos das prostaglandinas têm reduzido a \\
sensibilidade do mesmo.
\end{tabular}

Descritores: Glaucoma/diagnóstico; Soluções oftálmicas; Pressão intra-ocular; Agonistas de dopamina/uso diagnóstico; Sensibilidade e especificidade

\footnotetext{
${ }^{1}$ Oftalmologista do Centro de Referência em Oftalmologia da Universidade Federal de Goiás - UFGO - Goiânia (GO) - Brasil.

${ }^{2}$ Oftalmologista do Centro de Referência em Oftalmologia da UFGO - Goiânia (GO) - Brasil.

${ }^{3}$ Oftalmologista do Centro de Referência em Oftalmologia da UFGO - Goiânia (GO) - Brasil.

${ }^{4}$ Oftalmologista do Centro de Referência em Oftalmologia da UFGO - Goiânia (GO) - Brasil.

5 Oftalmologista do Centro de Referência em Oftalmologia da UFGO - Goiânia (GO) - Brasil.

Endereço para correspondência: Leopoldo Magacho Coordenador do Setor de Glaucoma do CEROF - UFGO Rua 131, número 313, Setor Sul, Goiânia (GO)

CEP 74093-200

E-mail: 1magacho@brturbo.com

Recebido para publicação em 29.03.2004

Versão revisada recebida em 20.07.2005

Aprovação em 02.12.2005

Os autores não possuem interesse comercial sobre qualquer produto mencionado no texto.

Nota Editorial: Depois de concluída a análise do artigo sob sigilo editorial e com a anuência do Dr. Humberto de Castro Lima sobre a divulgação de seu nome como revisor, agradecemos sua participação neste processo.
}

\section{INTRODUÇ̃̃̃O}

Vários testes foram estudados como indicadores prognósticos do glaucoma, como os testes de sobrecarga hídrica ${ }^{(1-2)}$, testes provocativos de midríase ${ }^{(3)}$ e ensaios terapêuticos ${ }^{(2)}$. Entretanto, ainda não existe um consenso sobre a real aplicabilidade dos mesmos na avaliação do paciente glaucomatoso.

Um novo teste foi incorporado ao nosso arsenal propedêutico. Estudos indicam que a ibopamina é útil na avaliação do glaucoma ${ }^{(4-7)}$, com sensibilidade e especificidades elevados no seu diagnóstico ${ }^{(7)}$. A ibopamina (3,4 diisobutirilester de N-metildopamina) é hidrolisada em epinina, um análogo da dopamina. Por sua vez, a epinina atua em receptores $\alpha$-adrenérgicos, causando uma midríase não cicloplégica, e em receptores D-1 dopaminérgicos, provocando um aumento na produção do humor aquoso ${ }^{(6,8)}$. Como vários subtipos de glaucoma são causados por uma dificuldade de drenagem no trabeculado, a ibopamina pode atuar como teste provocativo, já que teoricamente aumenta a pressão intra-ocular (Pio) de uma forma mais evidente em olhos glaucomatosos que em olhos normais. 
Entretanto, nos parece racional formular a hipótese de que o uso de drogas que levem ao aumento da drenagem do humor aquoso e/ou diminuam sua produção, pode influenciar o teste provocativo da ibopamina no glaucoma. Esse estudo foi desenvolvido para testar essa hipótese.

\section{MÉTODOS}

Trata-se de um estudo prospectivo comparativo. Os pacientes glaucomatosos foram consecutivamente recrutados no serviço de glaucoma do Centro de Referência em Oftalmologia (CEROF) da Universidade Federal de Goiás. Os indivíduos normais foram voluntários ou amigos e parentes dos pacientes. Consentimento livre e informado foi obtido de todos os participantes. Apenas um olho por paciente foi aleatoriamente incluído no estudo.

Os critérios de inclusão para os pacientes glaucomatosos foram: diagnóstico clínico de glaucoma primário de ângulo aberto (disco óptico apresentando diminuição localizada ou global da rima nervosa, gonioscopia revelando ângulo aberto, alteração vascular indicativa de glaucoma, como vasos em passarela ou baioneta, hemorragia de disco óptico e assimetria de escavação $>0,2^{(9)}$ ) comprovado por pelo menos dois exames perimétricos SITA Standard 24-2 (Humphrey Systems, Dublin, CA, EUA) confiáveis e reprodutíveis ${ }^{(10)}$. Os indivíduos foram excluídos caso apresentassem exame clínico compatível com qualquer entidade capaz de influenciar os resultados do campo visual ou da tonometria, como degeneração macular relacionada à idade ou cicatrizes corneanas, glaucoma secundário ou de ângulo estreito. Pacientes com história de qualquer cirurgia intra-ocular dentro de 6 meses prévios ao teste, ou cirurgia fistulizante também foram excluídos. Drogas hipotensoras em uso há pelo menos 6 meses foram registradas para todos os indivíduos incluídos no estudo.

A seguir, foram instiladas duas gotas de ibopamina $2 \%$ com um intervalo de 5 minutos. A Pio foi medida previamente em um tonômetro de aplanação de Goldmann (calibrado), e após 30, 60 e 180 minutos sempre pelo mesmo examinador e tonômetro. $\mathrm{O}$ examinador encontrava-se mascarado ao grupo do paciente. No nosso estudo, o teste da ibopamina foi considerado positivo quando a Pio excedeu $4 \mathrm{mmHg}$ em pelo menos uma das medidas.
A análise estatística foi realizada com o software SPSS (SPSS Inc. Chicago, IL, EUA), versão 10.1. Sensibilidade e especificidade do teste foram computadas, no grupo como um todo, e em seguir subdivididos de acordo com o uso de drogas antiglaucomatosas. O Teste $\mathrm{t}$ de Student Independente foi utilizado para comparar os grupos entre si e segregados de acordo com as drogas antiglaucomatosas em uso. O Teste t de Student Pareado foi utilizado para se comparar a Pio 30, 60 e 180 minutos após a utilização do colírio de Ibopamina. Valores de p menores que 0,05 foram considerados como significativos.

\section{RESULTADOS}

Cinqüenta e oito indivíduos (38 glaucomatosos e 20 normais) foram incluídos no estudo, sendo que 26 pacientes usavam $\beta$-bloqueadores, 8 usavam $\alpha$-agonistas, 8 utilizavam análogos de prostaglandinas e 5 inibidores tópicos da anidrase carbônica.

A distribuição da Pio antes do colírio de Ibopamina e após 30,60 e 180 minutos encontra-se disposta na tabela 1 . O aumento da Pio foi significativo aos 30, 60 e 180 minutos ( $\mathrm{p}<0,001$ ). A tabela $2 \mathrm{e}$ a figura 1 ilustram o aumento da Pio nos subgrupos. O aumento da Pio nos pacientes utilizando análogos das prostaglandinas foi estatisticamente inferior em relação aos pacientes que usavam outras drogas (Tabelas 1 e 2; Figura 2).

Finalmente, foram investigados somente os indivíduos com teste da ibopamina positivo em relação ao tempo das medidas da Pio. A positividade foi verificada em $96 \%$ dos pacientes aos 30 minutos, em $77 \%$ aos 60 minutos e em $42 \%$ aos 180 minutos após instilação da ibopamina. Em cada indivíduo do grupo normal com teste positivo da ibopamina, os valores da Pio retornaram ao nível basal 60 minutos após instilação do colírio.

Estipulando a elevação na Pio de mais $4 \mathrm{mmHg}$ como teste positivo, a sensibilidade foi de $68 \%$ e a especificidade de $95 \%$. Entretanto, ao se excluir os pacientes que utilizavam análogos das prostaglandinas, a sensibilidade aumentou para $87 \%$, sem alteração na especificidade.

\section{DISCUSSÃO}

A ibopamina $2 \%$ é um midriático não cicloplégico que aumenta a produção do humor aquoso ${ }^{(8)}$ induzindo hipertensão ocular em olhos glaucomatosos, onde o sistema de drena-

\begin{tabular}{|c|c|c|c|c|}
\hline Grupo & Pio prévia* & 30 minutos* & 60 minutos* & 180 minutos* \\
\hline Normal & $13,6 \pm 3,0$ & $15,0 \pm 3,6$ & $14,0 \pm 3,0$ & $12,3 \pm 2,3$ \\
\hline Glaucoma & $17,3 \pm 4,4$ & $23,8 \pm 6,7$ & $23,4 \pm 7,0$ & $20,4 \pm 6,2$ \\
\hline $\mathrm{p}^{\dagger}$ & 0,001 & $<0,001$ & $<0,001$ & $<0,001$ \\
\hline $\begin{array}{l}\text { Glaucoma (excluindo análogos } \\
\text { das prostaglandinas) }\end{array}$ & $18,2 \pm 4,5$ & $25,6 \pm 6,4$ & $24,8 \pm 6,2$ & $22,0 \pm 6,0$ \\
\hline Análogos das prostaglandinas & $14,2 \pm 2,5$ & $17,1 \pm 2,4$ & $18,0 \pm 5,2$ & $14,7 \pm 3,2$ \\
\hline $\mathrm{p}^{\dagger}$ & 0,02 & $<0,001$ & 0,007 & 0,002 \\
\hline
\end{tabular}




\begin{tabular}{|c|c|c|c|}
\hline Grupo & 30 min* $^{*}$ & $60 \mathrm{~min}^{*}$ & $180 \mathrm{~min}^{*}$ \\
\hline Normal & $1,4 \pm 2,4$ & $0,5 \pm 2,0$ & $-1,2 \pm 2,1$ \\
\hline Glaucoma & $6,5 \pm 4,8$ & $6,0 \pm 5,2$ & $3,1 \pm 4,7$ \\
\hline $\mathrm{p}^{\dagger}$ & $<0,001$ & $<0,001$ & $<0,001$ \\
\hline $\begin{array}{l}\text { Glaucoma (excluindo-se } \\
\text { análogos das prostaglandinas) }\end{array}$ & $7,4 \pm 4,8$ & $6,6 \pm 5,2$ & $4,3 \pm 5,3$ \\
\hline Análogos das & $2,8 \pm 2,6$ & $3,7 \pm 5,0$ & $0,5 \pm 3,5$ \\
\hline \multicolumn{4}{|l|}{ prostaglandinas } \\
\hline $\mathrm{p}^{\dagger}$ & 0,01 & 0,1 & 0,08 \\
\hline
\end{tabular}



Figura 1 - Variabilidade da pressão intra-ocular após a administração da ibopamina tópica a $2 \%$

gem geralmente se encontra prejudicado. Dessa forma, podemos formular a hipótese de que o aumento da Pio em indivíduos com glaucoma tende a ser maior que em olhos com o sistema de escoamento do humor aquoso dentro dos limites da normalidade. Conseqüentemente, é plausível tentar se estabelecer pontos de corte para esse aumento da Pio que leve a uma relação sensibilidade/especificidade elevada no diagnóstico do glaucoma. Neste estudo, o aumento da Pio foi consideravelmente maior em olhos glaucomatosos comparados aos indivíduos normais, confirmando a potencial utilização da ibopamina tópica a $2 \%$ como teste provocativo para o glaucoma.

$\mathrm{O}$ aumento da Pio acima de $4 \mathrm{mmHg}$ foi determinado para se considerar o teste positivo e os resultados deste estudo foram comparados favoravelmente com relatos prévios onde o ponto de corte do aumento da Pio foi de $3 \mathrm{mmHg}^{(4-5)}$. Uma possível explicação deve-se ao fato de termos medido a Pio em um intervalo de tempo menor à instilação da ibopamina (30 minutos vs. 45 minutos nos estudos prévios). Desta forma, conseguimos manter os mesmos níveis de eficácia do teste da ibopamina, porém com redução do seu tempo de duração. Este fator tornase importante pois, diminuindo-se ainda mais o tempo de per-

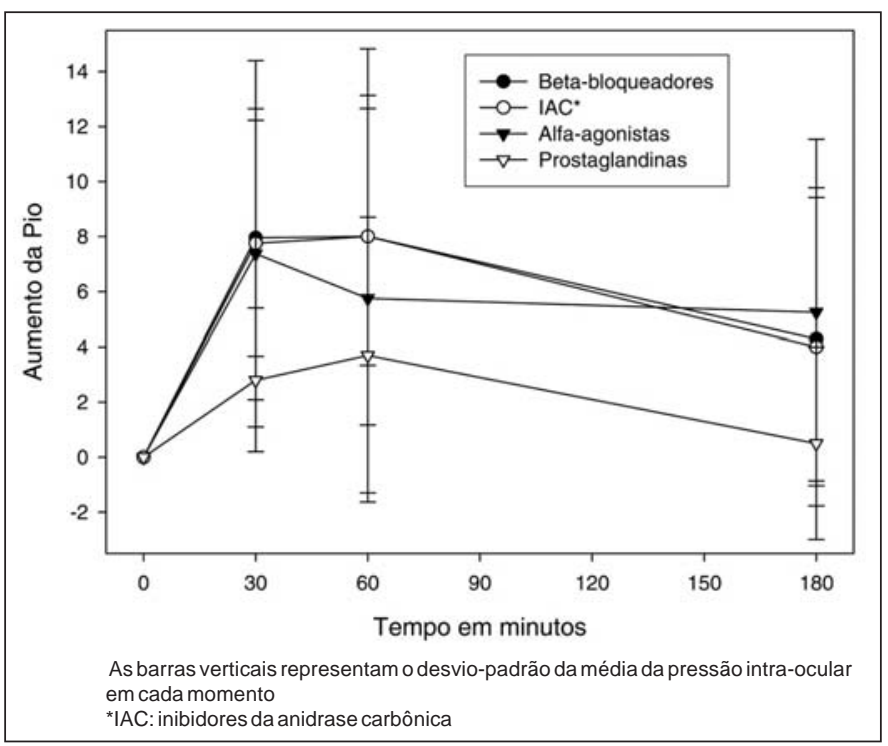

Figura 2 - Aumento da pressão intra-ocular após a administração da ibopamina tópica a $2 \%$ dividido de acordo com as medicações em uso

manência do paciente na clínica, pode-se realizar um número maior de exames por dia. Porque um fluxo trabecular normal não exclui glaucoma, não encontramos sensibilidade de $100 \%$ para o teste, e portanto os resultados falso-negativos podem ser esperados em baixas taxas ( $13 \%$ em nossa população).

$\mathrm{O}$ objetivo principal do presente estudo foi verificar se o teste seria útil em pacientes usando drogas antiglaucomatosas. Alguém poderia apontar que pacientes usando medicações hipotensoras deveriam ter o diagnóstico de glaucoma firmado e, com isso, não necessitariam de testes provocativos. Entretanto, é comum em serviços de referência em glaucoma pacientes em uso de anti-hipertensivos, mas sem diagnóstico firmado de glaucoma. Portanto, torna-se necessário o conhecimento da eficácia deste relativamente novo teste provocativo em tais pacientes, com o intuito de reduzirmos ainda mais aqueles que recebem tratamento antiglaucomatoso desnecessário. Todos os pacientes em uso de hipotensores tiveram uma resposta na Pio similar, exceto aqueles em uso de análogos das prostaglandinas (Figura 2). Nossos resultados diferem de um trabalho prévio ${ }^{(11)}$. Uma hipótese para o baixo desempenho do teste nesses indivíduos seria que o excesso de produção do humor aquoso pela ibopamina poderia ser drenada pela via alternativa úveo-escleral.

Como os pacientes estudados pertenciam a um serviço universitário de referência em glaucoma, a amostra foi desigual, ou seja, um maior número de usuários de beta-bloqueadores em relação às demais drogas. Tentamos diminuir o viés de inclusão selecionando os pacientes de forma consecutiva. Apesar disso, mesmo com o poder do teste diminuído devido a um número reduzido de pacientes utilizando análogos das prostaglandinas, foi notado que essa classe de drogas reduz o aumento da Pio, e com isso a performance do teste da ibopamina no glaucoma, limitando a utilidade do teste nestes pacientes. 
O único indivíduo normal com teste da ibopamina positivo retornou à normalidade 60 minutos após a instilação do colírio. Visitas posteriores indicarão se este é um teste falsopositivo, ou um paciente com deficiência na drenagem trabecular, que poderia levar ao glaucoma com o decorrer dos anos. Outro dado importante advindo do presente estudo, reside no fato de que a Pio retorna rapidamente para valores próximos àqueles prévios ao exame, reduzindo a chance de perdas adicionais de células do disco óptico da camada de fibras nervosas da retina, sobretudo em pacientes com glaucoma avançado.

\section{CONCLUSÕES}

O teste provocativo da ibopamina $2 \%$ pode ser uma ferramenta auxiliar no diagnóstico do glaucoma. O teste é rápido, barato, fácil de realizar e com alta sensibilidade e especificidade em populações glaucomatosas. Apesar da pequena amostra estudada, sugere-se que olhos em uso de análogos das prostaglandinas têm reduzido a sensibilidade do mesmo.

\section{ABSTRACT}

Purpose: To evaluate the ibopamine provocative test for the diagnosis of glaucoma in glaucoma patients using antiglaucomatous drugs. Methods: Two 2\% ibopamine eyedrops were instilled 5 minutes apart in one eye selected at random in both glaucoma and normal subjects. The intraocular pressure (IOP) was assessed prior to the drops and 30,60 and 180 minutes after instillation. The test was considered positive when there was an intraocular pressure increase of greater than $4 \mathrm{mmHg}$ at any one of the timepoints. The amount of intraocular pressure change was compared to the types of medical treatment. Results: Fifty-eight eyes were included ( 38 glaucoma patients and 20 normal individuals). The intraocular pressure rise was significantly higher in glaucoma patients ( $\mathrm{p}<0.001$ at all times). The sensitivity and specificity of the ibopamine test were $68 \%$ ( $87 \%$ if we exclude eyes using prostaglandin analogues) and $95 \%$, respectively. Glaucoma patients using prostaglandin analogues did not present a significant intraocular pressure elevation. Conclusion: The ibopamine provocative test may be an auxiliary test in glaucoma diagnosis. Despite the small sample size, concurrent use of prostaglandin analogues apparently reduces the test's sensitivity.

Keywords: Glaucoma/diagnosis; Opthalmic solutions; Intraocular pressure; Dopamine agonists/diagnostic use; Sensitivity and specificity

\section{REFERÊNCIAS}

1. Armaly MF. Water-drinking test. I. Characteristics of the ocular pressure response and the effect of age. Arch Ophthalmol. 1970;83(2):169-75.

2. Spaeth GL. Effects of topical dexamethasone on intraocular pressure and the water drinking test. Arch Ophthalmol. 1966;76(6):772-83.

3. Harris LS, Galin MA. Cycloplegic provocative testing. Arch Ophthalmol. 1969;81(3):356-8.

4. Lima FE, Guimarães NLD, Santos LM, Costa LP, Ávila M. Ibopamina tópica na propedêutica do glaucoma. Rev Bras Oftalmol. 2002;61(2):109-13.

5. De Gregorio F, Pecori Giraldi J, Pannarale L, Saccucci S, Virno M. Ibopamine in glaucoma diagnostics: a new pharmacological provocative test. Int Ophthalmol. 1996;20(2):151-5.

6. Virno M, Taverniti L, De Gregorio F, Sedran L, Longo F. Increase in aqueous humor production following D1 receptors activation by means of ibopamine. Int Ophthalmol. 1996;20(2):141-6.

7. Magacho L, Lima FE, Costa ML, Fayad FA, Guimaraes NL, Avila MP. Ibopamine provocative test and glaucoma: consideration of factors that may influence the examination. Curr Eye Res. 2004;28(3):189-93.

8. Virno M, Taverniti L, Pecori Giraldi J. Ibopamina: nuevo midriatico non cicloplegico (nota preliminare). Boll Ocul. 1986;65:1135-6.

9. Varma R, Spaeth GL. The optic nerve in glaucoma. Philadelphia: J. B. Lippincott; 1993.

10. Anderson. DR, Patella VM. Automated static perimetry. 2nd ed. St. Louis, Missouri: Mosby Year Book; 1999.

11. Pecori Giraldi J, Mollicone A, Seguto D, Simeone L, Zaccara F. Latanoprost and the ibopamine test: a year's

\section{ABO

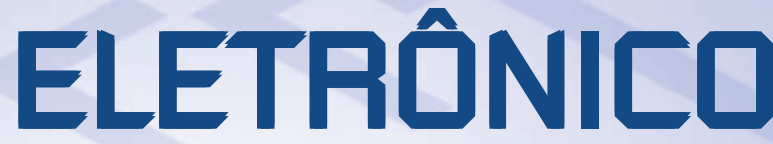 \\ A versão eletrônica dos Arquivos Brasileiros de Oftalmologia com textos completos está disponível em:}

- ABQ -

Arquivos Brasileiros de Oftalmologia

http://www.abonet.com.br

- 5cIELם - Scientific Electronic Library Online http://www.scielo.org

- Free Medical Journals - http://www.freemedicaljournals.com 